\title{
Properties of the "Orgamax" Osteoplastic Material Made of a Demineralized Allograft Bone
}

\author{
V. T. Podorognaya ${ }^{1, a)}$, I. A. Kirilova ${ }^{1, b)}$, Yu. P. Sharkeev ${ }^{2,3, c)}$, \\ P. V. Uvarkin, ${ }^{2, \mathrm{~d})}$, P. A. Zhelezny ${ }^{4}$, A. P. Zheleznaya ${ }^{4}$, \\ S. E. Akimova ${ }^{5}$, V. P. Novoselov ${ }^{6}$, and L. N. Tupikova \\ ${ }^{1}$ Novosibirsk Research Institute of Traumatology and Orthopedics n.a. Ya.L. Tsivyan, Novosibirsk, Russia \\ ${ }^{2}$ Institute of Strength Physics and Materials Science SB RAS, Tomsk, 634055 Russia \\ ${ }^{3}$ National Research Tomsk Polytechnic University, Tomsk, 634050 Russia \\ ${ }^{4}$ Novosibirsk State Medical University, Novosibirsk, Russia \\ ${ }^{5}$ Altai State Medical University, Barnaul, Russia \\ ${ }^{6}$ Novosibirsk Regional Clinical Bureau of a Forensic Medical Examination, Novosibirsk, Russia \\ a)VPodorognaya@niito.ru \\ b) Corresponding author: IKirilova@niito.ru \\ c)sharkeev@ispms.tsc.ru \\ d) uvarkin@ispms.tsc.ru
}

\begin{abstract}
We investigated properties of the "Orgamax" osteoplastic material, which was produced from a demineralized bone, in the treatment of extensive caries, in particular chronic pulpitis of the permanent teeth with unformed roots in children. The "Orgamax" osteoplastic material consists of demineralized bone chips, a collagen additive, and antibiotics. The surface morphology of the "Orgamax" osteoplastic material is macroporous, with the maximum pore size of $250 \mu \mathrm{m}$, whereas the surface morphology of the major component of "Orgamax", demineralized bone chips, is microporous, with a pore size of 10-20 $\mu \mathrm{m}$. Material "Orgamax" is used in the treatment of complicated caries, particularly chronic pulpitis of permanent teeth with unformed roots in children. "Orgamax" filling a formed cavity exhibits antimicrobial properties, eliminates inflammation in the dental pulp, and, due to its osteoconductive and osteoinductive properties, undergoes gradual resorption, stimulates regeneration, and provides replacement of the defect with newly formed tissue. The dental pulp viability is completely restored, which ensures the complete formation of tooth roots with root apex closure in the long-term period.
\end{abstract}

\section{INTRODUCTION}

Effective treatment of chronic pulpitis of the permanent teeth with unformed roots in children is based on the choice of an adequate treatment modality and an intervention limit that should ensure the maximum preservation of pulp viability and, at the same time, prevent the development of inflammation in tooth tissues [1-3]. The topicality and complexity of treating pulpitis of the permanent teeth with unformed roots make this issue one of the main challenges of practical pediatric dentistry because untimely and substandard treatment leads to premature loss of the teeth, disturbance of the normal development and relationship of the jaws, and also sensitization and intoxication of a child in general.

Preservation of viability of an inflamed tooth pulp is a topical issue. The tooth pulp feeds hard dental tissues, ensuring their sustained resistance. Healthy tooth tissues have sufficient strength and resistance. A tooth deprived of its pulp becomes less strong. Further, tooth tissues, enamel and dentin, become brittle, and the tooth is destroyed faster than a tooth with the viable pulp. During endodontic treatment, the tooth, as an organ, becomes weaker. To achieve a better operative approach during dental pulp extirpation, a significant amount of tooth tissues is removed by preparation. The tooth pulp is a barrier to the spread of infection into periapical tissues [3, 4]. Upon substandard treatment, teeth with pulp removed become foci of odontogenic infection in $20-84 \%$ of patients. 

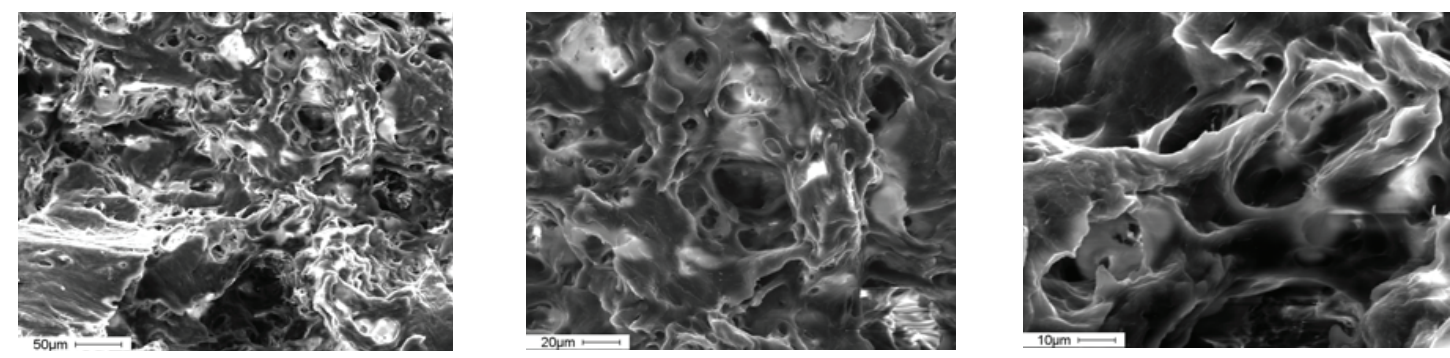

FIGURE 1. SEM images of the surface of DMBC

In children, $80 \%$ of all diseases of the odontogenic nature (inflammatory radicular cysts, odontogenic periostitis, and osteomyelitises) develop during the mixed dentition stage. These diseases are caused by complications of caries (pulpitis, periodontitis) resulting from incomplete and untimely sanitation.

Given the major global trends, a new osteoplastic material "Orgamax" [1-3] made of demineralized bone chips, a collagen-containing solution, and antibacterial drugs (cifran and axetin) was developed at the Laboratory of Preparation and Preservation of Biological Tissues of the Novosibirsk Research Institute of Traumatology and Orthopedics based on the authors' know-how [5].

In this regard, the study purpose was to explore the structural characteristics of the composite osteoplastic material "Orgamax", and its major component-demineralized bone chips (DMBC).

\section{MATERIALS AND METHODS}

The study material included the composite osteoplastic material, "Orgamax", and its major component-DMBC [5]. The osteoplastic material (OPM) was produced by compression, lyophilized, and sterilized by stream of fast electrons.

The surface morphology of bone materials was investigated by scanning electron microscopy (SEM) (Phillips SEM 515) with an EDAX attachment for microanalysis, which was also used to analyze the bone elemental composition.

The study included 10 children aged 6 to 12 years with chronic forms of pulpitis permanent teeth treated with "Orgamax" osteoplastic material. Methods: clinical and radiographic. The following 3, 6 and 12 months after surgery.

\section{RESULTS AND DISCUSSION}

Figure 1 shows SEM images of the surface of DMBC at different magnifications. The morphological structure of the surface of allogeneic DMBC is microporous, with a pore size of 10-20 $\mu \mathrm{m}$. Contours of DMBC chips are smoothed and slightly blurred, with projections and depressions (Fig. 1).

The surface of "Orgamax" OPM made of DMBC, a collagen additive, and antibiotics looks uneven, with projections and depressions (Fig. 2). Partly, the surface looks like a porous structure with the maximum pore size of up to $250 \mu \mathrm{m}$. Sometimes, the surface is twisted, with comb-like projections and smooth contours. It should be noted that the "Orgamax" material made of DMBC is an osteoplastic composite material because it is composed of three components $[5,6]$.
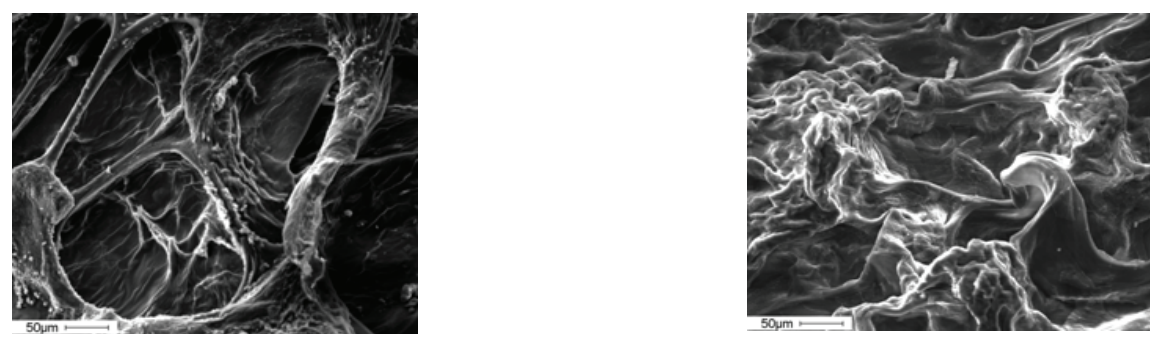

FIGURE 2. SEM image of the Orgamax OPM surface (sample TVCH 1060) made of DMBC 


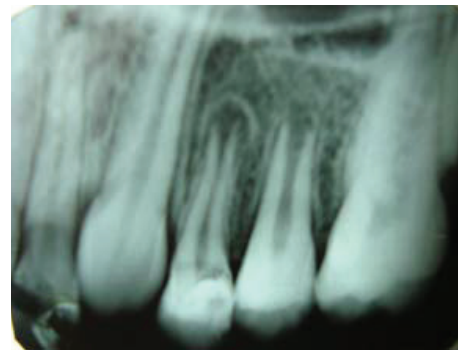

(a)

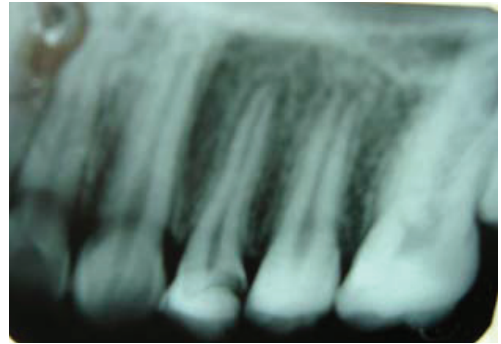

(b)

FIGURE 3. Patient $S$. Radiographic image of the 4th and 5th teeth. Diagnosis: chronic fibrous pulpitis of the 4 th and 5 th teeth. (a) before treatment; the tooth roots are not formed; (b) 1 year after treatment; the roots of the 4 th and 5 th teeth are formed, the root apices are closed

The osteoconductive properties and the ability to serve as a drug carrier vehicle are common to all materials on the basis of allogeneic bone fragments [5], while the "Orgamax" material additionally possesses the osteoinductive properties, which is confirmed by in vivo ectopic implantation tests [6].

The properties of allobone fragments subjected to demineralization, as a form of processing, make it possible to impart desirable characteristics to a composite material and to define OPM application features. "Orgamax" may be recommended for osteogenesis stimulation in the case of an unremarkable medical history.

The use of the developed composite osteoplastic material for reconstruction of bone defects eliminates the need in antibiotic therapy in the postoperative period because the material is a carrier of antibacterial drugs, provides their targeted delivery to the reconstruction area, and retains the drug concentration in the area in the postoperative period.

There is a high pulp amputation technique that uses a maximally deep resection of the root pulp in the root canal entrance $[3,4]$. The root pulp portion remaining in the canal is treated by medicines to stop the inflammatory process and to ensure further tooth root formation.

The disadvantage of the high amputation technique is the lack of root growth stimulation. Surgery eliminates inflammation, thereby ensuring continued growth of the root. Furthermore, this technique uses calcium hydroxidebased drugs [3,4]. Calcium hydroxide exerts the anti-bacterial and anti-inflammatory effects only on the superficial pulp layers that are in the immediate contact with the paste. However, the deeper layers receive no antibacterial treatment. Therefore, exacerbation of the inflammatory process in the root pulp requires more intensive antiinflammatory therapy. The wound is treated by mixtures of antibacterial agents having anti-inflammatory properties, e.g., a broad-spectrum antibiotic (neomycin, erythromycin, streptomycin, etc.) and enzymes (trypsin, chymotrypsin, chymopsin) with hydrocortisone emulsion or another glucocorticoid. In this case, antibacterial and antiinflammatory agents are repeatedly applied to the amputated pulp after 1-2 days. It should be noted that all these manipulations do not stimulate the tooth root formation.

To improve the efficacy of treatment of chronic pulpitis of the permanent teeth with unformed roots in children, a treatment technique was developed that includes formation of a cavity in the tooth crown, filling the cavity with a therapeutic composition, and tooth stopping [7]. The formed cavity is filled with the bioactive osteoplastic material "Orgamax" on the basis of an allogeneic demineralized bone matrix possessing the antimicrobial, osteoconductive, and osteoinductive properties. A temporary sealant is placed on the top. Then, the tooth condition is monitored for a year, including electrical conductivity measurement and radiographic control. In the case of positive changes demonstrating pulp viability, the temporary filling is replaced with a permanent sealant within 1-3 months.

The bioactive octeoplastic material "Orgamax" that has antimicrobial properties fills the formed cavity, eliminates inflammation in the dental pulp and, due to its osteoconductive and osteoinductive properties, undergoes gradual resorption, stimulates regeneration, and provides replacement of the defect with newly formed tissue. Fibroblasts synthesize collagen fibers whose architectonics reproduces the graft architectonics due to parallel processes of graft resorption and replacement. The osteoplastic material is gradually replaced with newly formed tissue rich in cellular elements. The reserve regenerative capabilities of the dental pulp make it possible to regenerate the tooth dentin under the influence of the osteoplastic material made of allogeneic DMBC. In this case, dental pulp viability is fully restored, which ensures the complete formation of tooth roots with root apex closure in the long-term period (Fig. 3). We present a clinical case of a 12-year-old patient S. According to a radiographic image of the 4th and 5th teeth, the patient was diagnosed with chronic fibrous pulpitis (Fig. 3a). The tooth roots are not formed, root apices have bell-shaped openings, growth areas are observed. The jawbone tissue around roots of the 4th and 5th teeth is not structured and has low contrast areas. According to an examination one year after 
treatment (Fig. 3b), the tooth roots are formed, and the apical openings are closed. Newly formed structured bone tissue is observed in the root apex area.

At the moment, according to the proposed method the operated on 10 children aged 6 to 12 years with chronic forms of pulpitis. In the study group patients were treated with the use of the OPM "Orgamax". After imaging and electro performed under anesthesia preparation cavity, antiseptic, cover the floor of the OPM "Orgamax" cavity filling glass ionomer cement. These electro-physiological point to recovery slurry parameters and restore the jawbone. Investigation continues.

Thus, osteoplastic properties of OPM "Orgamax" stimulate regeneration and provide the opportunity of the full formation of the roots of teeth in children with the closure of their tops with the structuring of the jawbone.

The advantages of the proposed technique are as follows:

- "Orgamax" may be used as a filling material;

- "Orgamax" is biocompatible with tissues of the tooth root, periapical bone, and body in general because material components are characterized by a high similarity to human tissues;

- the allogeneic material obviates antigenicity because it is subjected to appropriate processing (purification, lyophilization, radiation sterilization);

- the surface morphology of demineralized bone chips is microporous, with a pore size of 10-20 $\mu$, and the surface morphology of "Orgamax" made of these chips is macroporous, with the maximum pore size of up to $250 \mu \mathrm{m} ;$

- the porous structure imparts pronounced hemostatic properties to the material, which facilitates control of bleeding in the root canal;

- properties of the osteoplastic material made of the demineralized bone matrix enable three-dimensional root canal filling, not only in the main canal and parapulpar dentin;

- it preserves dental pulp viability;

- it retains the possibility of complete formation of the tooth roots with tooth apex closure.

\section{CONCLUSIONS}

Thus, the developed osteoplastic material "Orgamax" is a promising material for biological treatment of chronic fibrous pulpitis with unformed tooth roots in children. The porous structure of "Orgamax" enables three-dimensional root canal filling and imparts pronounced hemostatic properties to the material. The presence of antimicrobial drugs eliminates inflammation in the dental pulp and osteoplastic properties stimulate regeneration and provide the opportunity of the full formation of the roots of teeth in children with the closing of the tops of the roots with the structuring of the jawbone. Based on pre-clinical data we can predict a good clinical outcome. Investigation continues.

\section{ACKNOWLEDGMENTS}

The study reported in this article was conducted according to accepted ethical guidelines involving research in humans and/or animals and was approved by an appropriate institution or national research organization. The study is compliant with the ethical standards as currently outlined in the Declaration of Helsinki. All individual participants discussed in this study, or for whom any identifying information or image has been presented, have freely given their informed written consent for such information and/or image to be included in the published article.

\section{REFERENCES}

1. A. B. Fuks, Paediatr Dent. 30, 211-219 (2008).

2. Parisay, J. Ghoddusi, and M. Forghani, Iran Endod J. 10(1), 6-15 (2015).

3. P. A. Zhelezny, et al., Therapeutic Pediatric Dentistry Tutorial, Novosibirsk State Medical University, Novosibirsk Region, Assn. Dentists, Novosibirsk, 2007.

4. T. G. Petrova, P. A. Zhelezny, K. O. Samoylov, A. P. Zheleznaya, and S. E. Akimova, Endodontics Today 1, 36-39 (2012).

5. I. A. Kirilova and V. T. Podorozhnaya, RUS Patent No. 2344826 (24.04.2007), http://www.findpatent.ru/ patent/234/2344826.html.

6. I. A. Kirilova, Technologies Living Systems 5(2-3), 16-24 (2008), http://elibrary.ru/item.asp?id=11151338.

7. I. A. Kirilova, V. T. Podorozhnaya, L. N. Tupikova, and S. E. Akimova, RUS Patent No. 2566202 (17.10.2014), http://www.findpatent.ru/patent/256/2566202.html. 